\title{
Protée
}

\section{Démystification du signe et destruction du sens dans le kōan} zen

\section{Simon Kim}

Volume 39, numéro 2, automne 2011

URI : https://id.erudit.org/iderudit/1007168ar

DOI : https://doi.org/10.7202/1007168ar

Aller au sommaire du numéro

Éditeur(s)

Département des arts et lettres - Université du Québec à Chicoutimi

\section{ISSN}

0300-3523 (imprimé)

1708-2307 (numérique)

Découvrir la revue

Citer cet article

Kim, S. (2011). Démystification du signe et destruction du sens dans le kōan zen. Protée, 39(2), 55-63. https://doi.org/10.7202/1007168ar
Résumé de l'article

À la différence du zazen, la forme méditative du zen la plus connue, qui cherche à annihiler le rapport aux choses terrestres par le retrait dans la méditation (assise), le köan zen choisit d'emprunter aux " choses terrestres " pour mieux en libérer l'esprit. Le kōan zen, en effet, se caractérise par son recours au langage en des phrases qui ne font pas sens (du type «Qu'est-ce que la Voie ? " - « Oui. ») ; autrement dit, les « devinettes » et autres « historiettes absurdes » que propose le koan zen à ses pratiquants utilisent le signe, mais pour mieux le démystifier et défaire tous les liens qui le rattachent au sens. Il s'agit de dérouter non pas tant pour révéler l'absurdité ou le vide de toute pensée (langagière), mais surtout pour bousculer l'esprit dans son carcan de signification dans le but de l'amener à accéder au " non-moi (wu-wo) ", à la " non-pensée (wu-nian) » qui sont autant de définitions négatives de l'illumination bouddhiste. Si le monde est conçu comme un langage, pour emprunter la formule de Lacan, le kōan zen propose une sortie du monde par la destruction du sens, afin d'ouvrir l'esprit à la vérité du " non-mental (wu-nian) ", soit à l'éveil. On observera donc la stratégie du signe employée dans ces köan par les maîtres zen pour sortir l'esprit de ses habitudes.
Ce document est protégé par la loi sur le droit d'auteur. L'utilisation des services d'Érudit (y compris la reproduction) est assujettie à sa politique d'utilisation que vous pouvez consulter en ligne.

https://apropos.erudit.org/fr/usagers/politique-dutilisation/ 


\section{DÉMYSTIFICATION DU SIGNE ET DESTRUCTION DU SENS DANS LE KŌAN ZEN}

SIMON KIM

Bien que l'on puisse faire remonter l'histoire du zen ${ }^{1}$ à l'Inde du Bouddha historique et à son disciple Kasyāpa, c'est en Chine qu'il se développa et prit la forme qu'on lui connaît aujourd'hui. C'est notamment à Bodhidharma, le premier patriarche de la lignée chinoise du zen, que l'on doit d'avoir expliqué en quoi consiste le zen. Le gatha, ou quatrain, qui lui est attribué dit ainsi:

Une transmission spéciale hors des écritures

Fondée ni sur des mots ni sur des lettres;

Pointant directement l'esprit propre de l'homme,

Elle lui fait voir sa propre vraie nature et atteindre ainsi la bouddhéité.

(Dumoulin, 1988: 85; notre traduction [NT]) ${ }^{2}$

Appartenant au bouddhisme, le zen poursuit le même but: un éveil (bodhi) et une réalisation de soi (qualifiée d'une infinité de manières, mais qu'on appellera ici, par référence au quatrain de Bodhidharma, réalisation de sa propre «bouddhéité» ou "nature-de-Bouddha»). Le zen est donc une pratique, une "expérience» ${ }^{3}$ pour accéder à cet éveil, à la bouddhéité qui «pointe directement l'esprit propre de l'homme», c'est-àdire qui outrepasse la conscience ordinaire discriminante. Pour ce faire, le zen propose d'un côté d'annihiler cette conscience ordinaire (par la méditation assise ou zazen 4 , en japonais), et d'un autre côté de pulvériser les affects de l'esprit discriminant pour révéler «l'esprit propre de l'homme». Cette dernière manière de procéder est celle du kōan zen, la méditation (zen) sur des "cas" 5 .

Le quatrain de Bodhidharma nous apprend autre chose sur cette pratique du zen: c'est qu'elle est une "transmission" et que, par conséquent, elle s'inscrit dans un rapport de maître à disciple. De fait, dans la pratique du kōan zen, le maître soumet le kōan qu'il aura jugé approprié à son disciple et ce kōan servira à ce dernier de point de départ en même temps que de guide dans sa recherche. Ce qu'il convient de noter, toutefois, c'est que cette recherche n'est jamais purement intellectuelle. Si le zen est transmission, c'est une transmission qui n'est «fondée ni sur des mots, ni sur des lettres». Cette précision est également importante parce qu'elle révèle le caractère propre du bouddhisme zen: il n'enseigne pas, il "pointe du doigt». Dès le départ, l'accent est mis sur cette particularité d'un enseignement hors des écritures. Un kōan en soi n'apporte aucune certitude, aucune vérité sainte, mais il est porteur de ces vérités. 
C'est bien la raison pour laquelle les adeptes du kōan zen donnent leur préférence à ces kōan plutôt qu'aux textes sacrés - les sūtras et leurs commentaires. En effet, le kōan est le lieu possible de l'expérience zen, quand les textes canoniques restent à la périphérie de cette expérience, se bornant à la décrire ou à la commenter. La littérature zen des kōan regorge d'ailleurs d'épisodes ou de "cas» dans lesquels les textes canoniques, les sūtras, sont rejetés, dédaignés et rabaissés. Isshū Miura nous fait remarquer que, à plusieurs reprises dans son traité Sokkō roku kaien fusetsu, le maître zen japonais Hakuin tient des propos virulents contre les enseignements sacrés; ainsi, dit-il à ses lecteurs, «votre attitude à l'égard des enseignements verbaux des bouddhas et des patriarches doit être aussi hostile qu'envers un ennemi mortel» $\left(1965: 46 ;\right.$ NT) ${ }^{6}$. Et D.T. Suzuki ne relate-t-il pas dans ses Essais sur le bouddhisme zen l'histoire de l'érudit De-shan qui, à la suite de son éveil, brûla les commentaires du Vajracchedikā Sūtra qu'il étudiait depuis plusieurs années (1972, vol.2: 41-42)? Cette "hostilité» ne doit pas être prise à la lettre 7 , mais elle témoigne néanmoins du fait que la réalisation de soi, de sa propre bouddhéité, ne peut se faire par voie de concept, mais «doit sortir des profondeurs de la conscience de chacun, indépendamment de l'enseignement des Écritures ou de l'aide de quelqu'un d'autre» (ibid., vol.1: 111).

Il n'empêche que cette transmission qui prétend n'être «fondée ni sur des mots, ni sur des lettres» a pourtant bien recours à des textes, aux kōan, qui sont constitués de mots et de lettres. Tout en rejetant le langage, elle n'a d'autre moyen que de recourir au langage pour nous amener à l'éveil de la réalisation de soi. On pourrait avancer un premier élément d'explication de ce paradoxe en évoquant ce kōan cité par le maître zen japonais Dōgen dans son œuvre Shōbōgenzō:

Un jour, un des moines du Grand Professeur Kōkaku du Mont Ungo lisait un sütra dans sa chambre. Le maître l'appela par la fenêtre: "Quel sūtra lisezvous?" Le moine répondit: «Le Vimalakìrtinirdesa Sūtra.» Le maître dit: "Je ne parlais pas du sūtra que vous tenez dans vos mains. Quel sūtra êtes-vous en train de lire?» Le moine fut soudain illuminé. $(1977,2: 12 ; \mathrm{NT})^{8}$
Ce qui est en jeu dans le kōan, ce ne sont ni les mots, ni les lettres, mais ce que justement les mots et les lettres empêchent de saisir. Le kōan zen va donc se servir du langage pour mieux révéler ses limites, son incapacité à sortir de sa fonction discriminante (puisqu'en disant qu'une chose est telle, je dis qu'elle n'est pas autrement) et discursive. Pour ce faire, le kōan zen utilise toutes les ressources imaginables de démystification du signe et de destruction du sens pour nous contraindre à

[...] abandonner les structures rationnelles de notre pensée et

à nous placer en deçà de notre état usuel de conscience de manière à pénétrer des dimensions nouvelles et inconnues.

(Dumoulin, 1988: 246; NT) ${ }^{9}$

Ce travail du kōan zen sur le langage s'attaque avant toute chose au langage discursif, nous l'avons dit. En tant qu'enseignement, en tant que transmission, le kōan zen se distingue précisément des textes canoniques en ce qu'il rejette leur discursivité. Un discours, en effet, est toujours discours sur, et donc impuissant à provoquer l'«expérience» que veut être le zen. Le zen, en ce sens, ne saurait être dogmatique. Nombre de kōan mettent en scène des maîtres invités à faire des sermons autour des sūtras ou de la doctrine du bouddhisme. Mais ces «sermons» se refusent systématiquement à être des discours, comme l'illustre fort bien cet épisode tiré des Entretiens de Lin-Tsi:

«Si je monte sur cette chaire aujourd'hui, c'est que je ne puis faire autrement ${ }^{10}$ - c'est par respect humain. Pour prôner notre Grande Affaire, si je m'en tenais à la tradition de notre lignée de patriarches et de disciples, je n'ouvrirais simplement pas la bouche, et vous n'auriez où mettre le pied. Mais, en prenant en considération la prière instante de Monsieur le conseiller ordinaire, comment en ce jour pourrais-je cacher mes principes? Y a-t-il donc quelque habile général qui soit prêt sur-le-champ à disposer ses troupes et à déployer ses étendards?

Qu'il témoigne devant l'assemblée, pour voir!»

Un moine demanda quelle était la grande idée du bouddhisme. Le maître fit khät ${ }^{11}$. Le moine s'inclina. Le maître dit: "En voilà un qui se montre capable de soutenir une discussion». (Demiéville, 1972: 21-26)

Ainsi que nous l'apprend ce passage, la «tradition de notre lignée de patriarches et de disciples", en 
matière de sermon et de discours, consiste à «ne pas ouvrir la bouche» ${ }^{12}$. C'est à tel point que Fong You-Lan, dans son Précis d'histoire de la philosophie chinoise, présente le zen comme une "philosophie du silence» (1992: 264). Toutefois, l'exemple de Lin-Tsi nous apprend autre chose sur le fonctionnement du kōan: s'il rejette la discursivité du sermon, le maître zen privilégie l'échange avec un moine, le dialogue. Encore une fois, puisqu'il est transmission, il faut qu'il y ait un rapport, une relation de maître à disciple qui s'établisse; et c'est à l'intérieur de cette relation que le kōan va opérer. Hui-neng, dans son Sütra de l'Estrade (cas unique dans la littérature zen d'un texte «théorique» sur la pratique du zen), écrit ainsi:

Amis dans le bien, l'homme est naturellement, tout au fond

de lui-même, riche de la sagesse de la bodhi et de la prajna.

Cependant, son esprit erre au gré des circonstances et il

ne peut s'en rendre vraiment compte par lui-même. C'est

pourquoi il doit recourir à un grand ami dans le bien qui lui

montrera la Voie et lui fera voir son essence.

(Fa-hai, 1995: 30)

Dans le cadre d'une telle relation, une forme de communication intervient qui appelle à nouveau le langage ou la parole. Mais quelle espèce de communication non discriminante et non discursive le kōan zen va-t-il pouvoir mettre en ouvre? En effet, le dialogue du kōan zen est bien loin de la dialectique définie par Platon comme «art d'interroger et de répondre» (390c), car souvent la réponse ne présente aucune connexion rationnelle avec la question ${ }^{13}$. C'est que, jusque dans le dialogue, le kōan zen fuit ce qui pourrait être une pensée discriminante et discursive. Il semble qu'intervient également à ce stade quelque chose de spécifique au chinois - et qui explique en partie que le kōan zen se soit tant développé en Chine - qui relève de sa stratégie d'énonciation que François Jullien résume en ces termes: «au lieu qu'il se déploie en logos, sa vertu est d'être indiciel» (1995: 228). Autrement dit, dans la tradition chinoise, «dire une chose, c'est la détacher du silence, se prononcer sur son importance, la souligner» (ibid.: 112). Par le biais de l'indiciel, l'interlocuteur est sollicité à un autre niveau de conscience que celui de la dialectique platonicienne.
Si la question posée est la preuve d'une recherche, c'est une recherche intellectuelle que l'indiciel déçoit:

Qu'est-ce que le zen? - C'est cela. - Qu'est-ce que la Voie? -

Oui. (App, 1994: 106; NT) ${ }^{14}$

Mais en même temps il oriente la recherche sur un plan plus spirituel - en tout cas non intellectuel, non discursif - qui sera, au bout du compte, celui de la réalisation. En effet, l'indiciel cultive la «vertu d'inachèvement d'une parole qui laisse d'autant plus à penser qu'elle commence seulement à dire et, par sa dispersion, fait échec au dogmatisme» (Jullien, 1995: 251). On parle alors d'un semina dicendi, d'une parole qui féconde la conscience. Toutefois, quand dans le dialogue confucéen la réponse est dite "indicielle», elle reste l'indice de quelque chose; autrement dit, elle «soulève un angle» qui doit permettre à l'interlocuteur de trouver en retour les trois autres. Suivant cet art du dialogue chinois, il doit suffire, pour citer Confucius, "d'entendre expliquer une chose pour en comprendre dix». Dans le kōan zen, en revanche, le coin soulevé ne révèle que du vide, il n'est l'indice de rien, à l'image du «cyprès dans la cour» de Zhao-zhou. La stratégie sur laquelle elle repose est cependant la même: il s'agit de fait de mettre l'interlocuteur dans la position mentale de s'ouvrir aux possibilités offertes par l'indiciel, par le «coin soulevé».

En réalité, l'indice du kōan zen, ce qu'il cherche à montrer sous le coin soulevé, c'est l'inanité du langage lui-même. Le dialogue est donc nécessaire pour «soulever un coin» et révéler qu'il n'y a rien qui puisse être dit:

Les mots ne peuvent exprimer les choses;

Le langage n'est pas conforme.

Ballotté par les mots, on est perdu;

Retenu par les phrases, on est désorienté.

(Aitken, 1991: 227 ; NT) ${ }^{15}$

Si le langage n'est pas conforme, c'est qu'il fixe, qu'il «réifie» ontiquement ce qu'il dit; le langage donne une valeur aux choses qu'il désigne, valeur qui est prise à tort pour une valeur ontologique. Ainsi, l'Avatamsaka Sūtra affirme que «la vérité n'a rien à faire avec les mots; elle est bien au-delà d'eux" (Suzuki, 1972, vol.2: 12). Les maîtres zen, dans leur 
acharnement à dénigrer tous les affects de la parole, veulent ainsi mettre l'accent sur ce trait spécifique du langage. Il s'agit toujours de veiller à ne pas prendre les mots pour les choses qu'ils désignent. C'est ainsi que Lin-Tsi explique: "Vous ne pensez qu'à chercher le Bouddha: le Bouddha est un nom" (Demiéville, 1972: 67). Le langage, lorsqu'il nomme une chose, l'appelle à une présence, en l'arrachant au silence de l'indifférencié. Ainsi, le langage instaure une différence; une différence qui n'est pourtant pas celle de l'objet et du sujet, puisque le nom reste un nom - et non l'objet qu'il signifie. La différence qui se fait jour, c'est la différence du signe, qui vient introduire un plan de présence ni purement objectif, ni purement subjectif. Jean Beaufret dit à ce sujet que la langue est, "plus qu'un simple moyen, l'apparition d'un monde qui est l'entre-deux du sujet et de l'objet" (1974: 71). Ce «monde» est donc un monde sans existence, mais qui parle de l'existence, de l'être (nous avons parlé de présence). Aristote va jusqu'à généraliser cette parole sur l'être; en effet, par son recours systématique au schéma prédicatif, il souligne l'omniprésence de l'être dans le langage. Par ailleurs, cette structure prédicative du monde, que la langue fait apparaître, présente les objets qu'il comprend sous une forme le plus souvent définitionnelle. Et la définition est bien la formule qui exprime le mieux l'essence des choses, ainsi que le remarque Aristote dans ses Topiques: "Ce qui signifie l'essence a pour nom définition» (I,4: 101b). Notons toutefois que, dans le cas du chinois - et par voie de conséquence du kōan zen -, le langage «n'envisage pas le réel en termes d'“être" (opposé au devenir)» (Jullien, 1995: 264). En effet, le chinois n'est pas une langue copulative; le verbe «être» (shi 是) est un terme qui n'a pas cette valeur singulière qu'il peut avoir dans les langues indo-européennes. Shi signifie, comme nom, la "vérité»; comme adjectif, "vrai»; et comme verbe, "être», "considérer comme véritable» ou encore «affirmer» (Couvreur, 1966: 422). Ainsi, si dans le schéma prédicatif de la définition, on trouve parfois shi en place de copule, le plus souvent cette copule est absente. Cela tient au fait que nombre de caractères chinois dénotent autant un nom qu'un verbe et que l'adjectif chinois contient sa copule ${ }^{16}$.
Par ailleurs, il faut encore remarquer qu' «être» en français sert souvent à rendre, en plus du shi, un autre caractère, you (有), qui signifie littéralement "avoir». Le you chinois est l'être de l'existence. On voit donc que la réalité est de fait envisagée en chinois non pas comme essence, mais sur le mode de l'avoir, de l'appartenance. «Être» et "non-être», dans le taoïsme et dans le zen, c'est you et wu (無), «avoir» et "nonavoir». On se trouve alors face à un discours qui n'est plus le discours définitionnel et prédicatif qui cherche à figer dans un nom, mais un discours de l'avoir, c'est-à-dire un discours qui cherche à épouser le mouvement d'une réalité conçue non plus comme étant ceci ou cela, mais comme ayant ou n'ayant pas tel attribut. Dès lors, le langage s'articule en actions, dans le passage d'un attribut à un autre, d'une forme à une autre. C'est ce qui permet à Marcel Granet de dire qu'en chinois «la parole est acte»: «le mot ne cesse pas d'évoquer une action et demeure foncièrement verbe» (1968: 38).

Il n'en demeure pas moins que, pour reprendre Jacques Lacan, «les choses du monde humain sont des choses d'un univers structuré en paroles, le langage, [...] les processus symboliques dominent tout, gouvernent tout» (1990: 57). Ainsi, lorsque les maîtres zen utilisent le paradoxe et le non-sens, c'est pour désarticuler cette structure langagière de l'univers. C'est aussi pour cette raison qu'ils privilégient à ce point le silence; en ce sens, le début du deuxième kōan du Bi Yan Lu est révélateur:

Zhao-zhou parla devant l'assemblée et dit: «La Voie authentique n'est pas difficile. Elle évite simplement choix et attachement. Un seul mot peut suffire à provoquer choix et attachement.» (Sekida, 1977: 149; NT) ${ }^{17}$

Ainsi, parler, c'est choisir un attribut et l'attacher à la réalité, mais c'est aussi s'attacher à cette réalité faussée par la détermination du langage, ce qui est contraire à la Voie ultime. C'est dans ce sens encore qu'il faut comprendre ce nouveau «sermon" de LinTsi:

Tout ce que vous rencontrez, au-dehors et (même) au-dedans de vous-même, tuez-le!

Si vous rencontrez un bouddha, tuez le bouddha!

Si vous rencontrez un patriarche, tuez le patriarche! 
Si vous rencontrez un arhat, tuez l'arhat!

Si vous rencontrez vos père et mère, tuez vos père et mère!

Si vous rencontrez vos proches, tuez vos proches!

C'est là le moyen de vous délivrer et d'échapper à l'esclavage

des choses; c'est là l'évasion, c'est là l'indépendance!

(Demiéville, 1972: 117)

"Tuer tout ce qu'on rencontre», c'est s'affranchir de l'attachement à tout ce que le langage (et la pensée) détermine. "L'esclavage des choses», c'est tout aussi bien «l'esclavage du langage». De fait, sachant que «l'esprit est toujours lesté de langage» et que ce dernier «est le système de différenciations dans lequel s'articule le rapport du sujet au monde» (MerleauPonty, 1968: 37), on comprend que le kōan zen cherche à rompre ce rapport qui obstrue l'accès à la réalisation de soi, de sa bouddhéité qui n'est autre que l'annihilation du moi ${ }^{18}$ comme sujet par la sortie hors du monde.

Ce qui désarçonne le lecteur d'un kōan, c'est donc bien que

[...] les kōan utilisent des signes et des signifiants décentriques

- on pourrait dire que les kōan eux-mêmes représentent le

processus de décentrement de tous les signes - de sorte qu'ils

fonctionnent avec flexibilité et provisoirement comme un

discours symbolique sans référence à un signifié absolu ou transcendantal. (Heine, 1994: 20; NT) ${ }^{19}$

Si l'on devait trouver un sens à ces kōan, ce serait donc dans le décentrement du signe qu'il faudrait le chercher; c'est ce que nous avons appelé la démystification du signe, puisqu'il s'agit de sortir de la croyance "mystifiante» que le signe pointe vers un signifié, une réalité, un être. Pour le zen, il n'y a ni être ni non-être, nous l'avons vu; tout au plus peut-on parler, avec Deleuze, d'un «extra-être» qui ne prétend même plus faire sens, qui est un vide: «à travers les significations abolies et les désignations perdues, le vide est le lieu du sens ou de l'événement qui se compose avec son propre non-sens, là où n'a plus lieu que le lieu" (1969: 162). Le vide est d'ailleurs ce qui qualifie le mieux, ou au plus près, la réalité du bouddhisme zen.

Le vide, gong (空) en chinois, traduit le concept indien de śūnyatā (vide ou vacuité) qui est au cœur de l'école dite du Milieu (Mādhyamika) animée par Nāgārjuna. Le vide, ici, n'est pas ce qui s'opposerait à un plein; il est, à l'instar du wu (non-avoir), son synonyme, au-delà de la dichotomie être/non-être. Il est même très précisément le lieu où le monde peut être un non-monde, le «lieu du sens qui se compose avec son propre non-sens». C'est dans ce sens qu'on peut trouver, dans le $X_{i n} X_{i n}$ Ming, cette caractérisation du vide comme lieu privilégié de la pensée:

Dans le vide unique les deux s'assemblent

Il contient également les Dix mille formes.

(Seng Ts'an, 1992: 23; NT) ${ }^{20}$

Le danger, on le voit bien, réside dans la tentation de réifier le vide en le définissant ou même en ne le définissant pas, par le simple fait qu'on le nomme (on pourrait très bien reformuler ainsi l'avertissement de Lin-Tsi: «Vous ne pensez qu’à chercher le Vide: le Vide est un nom»). Ni Nāgārjuna, ni les maîtres zen n'en sont dupes; Nāgārjuna dans son Madhyamakakārikā écrit de fait que la vacuité (śūnyatā) est une désignation métaphorique, une vérité de pure «convention» (Garfield, 1995: 68) ${ }^{21}$; et Shen-hui d'expliquer que "c'est pour ceux qui n'ont pas encore pénétré leur nature propre que l'on parle de vacuité. Si l'on voit sa nature foncière, la vacuité elle-même n'existe pas» (Gernet, 1977: 43). Le vide est le lieu où s'absorbent toutes les significations, où disparaît le sens; il est la métaphore de ce qui ne peut être dit que par le biais de la métaphore ou du paradoxe 22 .

Lieu où s'annulent les dualismes, les discriminations, le vide désigne surtout ce que Deleuze appelle «le lieu où l'immédiat se tient “immédiatement" comme non-à-atteindre» (1969: 162); autrement dit, le lieu ou le moment de l'expérience ultime du zen, celle de la réalisation de sa nature propre ou de sa bouddhéité, où ce qui est à atteindre est là comme "non-à-atteindre». Si le zen n'est pas un enseignement, s'il est sans doxa, c'est parce qu'il est transmission non pas d'une vérité intelligible, mais de cette expérience ultime qui ouvre l'esprit au vide, à «l'immédiateté du non-à-atteindre», ou plus exactement qui annihile l'esprit dans cette immédiateté: 
Le moment viendra où toutes pensées cesseront de se lever, et il n'y aura plus de travail de la conscience. C'est alors que vous briserez votre cerveau en morceaux et pour la première fois comprendrez que la vérité est en votre possession depuis le premier commencement. (Suzuki, 1972, vol. 2: 92)

Ce moment «où toutes pensées cesseront de se lever» est précisément ce que Deleuze appelait un lieu; c'est ce lieu d'immédiateté que Sekida traduit par «direct pure cognition» (pure cognition directe) ${ }^{23}$. Cette traduction ne fait que dire autrement ce rejet de la médiation (du langage et de la pensée) pour accéder directement (im-médiatement) à cette vérité qui devient partout perceptible pour celui qui a débarrassé son cœur de l'esprit discriminant «lesté de langage». Et si le terme de lieu est préférable à celui de moment pour parler de ce point où l'expérience se produit enfin, c'est que le moment est un moment figé (ce moment précis où...), tandis que le lieu reste ouvert à ce qui s'y déroule. De fait, le moment renvoie à la pensée discursive qui correspond au caractère chinois nian (念). Or, ce caractère est lui-même composé de deux idéogrammes, xin (心) d'un côté, et de l'autre jin (今) qui signifie «maintenant», le «moment présent». Ainsi, la pensée discursive (que Suzuki traduit parfois par «le mental», soulignant son caractère opératoire et discriminant) est actualisation (jin) de l'esprit non discursif (xin). Elle marque sa venue au temps, à la présence; elle fixe un moment du flux continu de l'esprit.

L'esprit non discursif, le xin, c'est aussi le cour représenté par cet idéogramme (心). En fait, il s'agit d'«un des termes les plus significatifs et les plus vastes aussi bien de la philosophie chinoise que dans le chinois courant» (Suzuki, 1972, vol.3: 109). Comme "cour", il est l'organe central, le siège de la vie, c'est-à-dire de tout ce qui nous détermine en tant qu'êtres humains vivants; dès lors, xin sera tour à tour le "cœur» comme siège des affects, l'«esprit» comme siège de l'intelligence, et même l' "estomac" comme élément vital de notre système physiologique. Situer la pensée vitale du xin dans le cœur, et non pas dans la tête, c'est la distinguer de la pensée-intellection ou discursive et discriminante. C'est ainsi que le kōan zen identifiera le xin au wu nian (無念), autrement dit à «l'absence de pensée» ou à la «non-pensée» - que Suzuki traduit par le «non-mental». Cet esprit non mental qui refuse de se fixer dans l'instant, dans le maintenant, est en prise directe et immédiate avec la réalité du Vide, puisqu'il n'a pas recours à la médiation de la pensée et à sa discursivité articulante. Il saisit au contraire le réel en deçà de toute articulation, dans son indétermination linguistique et conceptuelle. L'expérience dont parle le kōan zen, c'est donc la cognition, la réalisation que notre esprit non mental participe de tout temps - hors d'un maintenant discriminant - de ce lieu "où l'immédiat se tient "immédiatement" comme non-à-atteindre".

Expliquer comment fonctionne le kōan zen, observer comment il détourne le processus de signification pour aboutir au vide comme réalité ultime, parler, enfin, de l'immédiateté de l'expérience de réalisation de sa nature propre ne permettent pas de provoquer cette expérience. Pour reprendre l'explication de Shen-hui que nous avons déjà évoquée, «c'est pour ceux qui n'ont pas encore pénétré leur nature que l'on parle de vacuité»; la véritable transmission, elle, se fait de cœur à cœur, d'esprit à esprit (de xin à xin), directement, sans passer par le langage et la pensée discursive. Le maître Dōgen parle à plusieurs reprises de «transmission directe, face-à-face» qui ne peut s'accomplir que de bouddha à bouddha (yuibutsu yobutsu). Certes, la nature-debouddha (ou bouddhéité) étant en chaque individu, Dōgen n'exclut a priori personne de cette transmission. Et pourtant, il s'agit bien, comme le soulignait le quatrain de Bodhidharma, d'une transmission spéciale. Comment donc comprendre cette spécialité de la transmission? Évoquons ici l'épisode qui fonde la tradition zen dans le bouddhisme indien et que relate Wu-men, le compilateur des kōan du Wu Men Guan:

Un jour, aux temps anciens, alors que le Très Honoré était sur le Mont Grdhrakūta, il brandit une fleur devant son assemblée. Tous étaient silencieux. Seul Mahākāshyapa [Grand Kāshyapa] se fendit d'un sourire. Le Très Honoré dit: «J'ai le Vrai CEil du Dharma, l'Esprit Merveilleux du Nirvana, la Vraie Forme du Sans-Forme, et la Subtile Porte du Dharma qui ne repose sur aucun mot ni aucune lettre, mais qui est une transmission spéciale hors des écritures. Ceci, je le remets à Mahākāshyapa». (Aitken, 1991: 46; NT)24 
Quelque chose s'est produit, un lieu d'immédiateté s'est ouvert alors que le Bouddha Shākyamuni était en compagnie de tous ses disciples. Mais seul Kāshyapa reçoit la «transmission spéciale hors des écritures». L'épisode, en soi, est structuré comme les autres kōan sous la forme d'un dialogue: le maître (ici, le Bouddha Shākyamuni) pose une question (il brandit une fleur) et le disciple (Kāshyapa) répond (il se fend d'un sourire) et révèle par sa réponse sa réalisation intérieure. De tous les disciples présents dans l'assemblée, un seul «comprit» ou fit l'expérience de la réalisation de sa nature-deBouddha. Ce que nous apprend cet épisode, c'est que non seulement cette transmission est directe, mais surtout qu'elle n'est spéciale que pour celui qui la reçoit, c'est-à-dire qui est prêt à la recevoir. Quand le disciple n'est pas prêt, les maittres zen le soulignent par des remontrances physiques ou verbales. C'est la spécialité de certains grands maîtres tels que Ma-zi, Lin-Tsi ou Huang-po que de sanctionner chaque signe d'hésitation de la part d'un moine par une gifle. La sanction tombe parce que l'hésitation marque la présence de la réflexion; elle est le signe d'une activité persistante de l'esprit discriminant et la preuve que le disciple n'est pas prêt.

Le processus du kōan zen requiert donc de la part des deux interlocuteurs - le maitre et le disciple - un état particulier (correspondant à l'état de samādhi dans le bouddhisme indien) qui doit être, chez le disciple, un état de questionnement avancé. On trouve un schéma similaire dans l'enseignement confucianiste que François Jullien décrit ainsi:

C'est seulement quand l'esprit de l'interlocuteur est prêt que

le maître l'éclaire par l'indication attendue. Peu de mots

suffisent alors; plutôt que de prodiguer sa parole, le Maître

se contente de donner un coup de pouce. Mais parce qu'elle

rencontre alors une disponibilité maximale de la part de

l'auditeur, que celui-ci est motivé, cette incitation est décisive,

elle réussit à débloquer en lui ce qui l'empêchait de progresser.

(1995: 232)

L'indiciel du langage ne peut ouvrir sur ce dont il est indice que si l'auditeur est en mesure de comprendre cet indice et de le suivre jusqu'au bout. Cet état de questionnement avancé, de «disponibilité maximale», est poussé à sa limite par l'a-rationalité du kōan, par son refus d'être une réponse articulée. Ainsi, lorsqu'il n'était qu'un jeune moine, LinTsi, nous apprend D.T. Suzuki, avait poussé son questionnement si loin que

[...] son esprit n'était qu'un grand point d'interrogation sans objet défini; et tel était son esprit, tel était l'univers: un point d'interrogation qu'il ne savait même pas au bout de quelle question placer, car il n'y avait encore nulle part rien de défini. [...] De fait, c'est à cause de cet état d'esprit qu'il ne savait quelle question définie il pouvait apporter devant son maître. (1972, vol. 2: 51)

Dans un tel état de désespoir mental, les coups que lui infligea son maître Huang-po eurent cet effet de "coup de pouce» ou de «déclic» qui provoqua la crise libératrice, c'est-à-dire l'expérience de réalisation de sa nature propre. Tout kōan entend fonctionner comme les coups de bâton de Huang-po sur son disciple; il vise à provoquer la crise libératrice. La démystification du signe et la destruction du sens en œuvre dans le kōan conduit l'esprit du disciple qui est en pleine détresse mentale devant une impasse et l'incite à «lâcher prise»: «l'arrivée à un cul-de-sac est en réalité le vrai point de départ de l'étude du zen", dit ainsi Suzuki (1964: 108; NT) ${ }^{25}$. Autrement dit, le non-sens du kōan est un cul-de-sac pour la pensée discursive du nian et une invitation à s'ouvrir à la pensée non mentale du xin; le "lâcher-prise» est un saut volontaire dans le vide. L'esprit, mis au bord du précipice par l'a-rationalité percutante du kōan, est invité à faire un pas de plus, à «briser le cerveau en morceaux", à annihiler tout ce qui se présente au-dehors et au-dedans de la conscience qui est dès lors définie ainsi dans le Sütra de la grande sagesse (Prajñāāāramitā Sūtra):

La conscience, c'est le vide, et le vide, c'est la conscience; le vide n'est pas différent de la conscience et la conscience n'est pas différente du vide. Quoi que la conscience soit, c'est le vide. Quoi que le vide soit, c'est la conscience.

(Merton, 1995: 138) 


\section{NOTES}

1. Zen est la prononciation japonaise du chinois chan qui est lui-même une transcription plus ou moins phonétique du sanskrit dhyāna. Par commodité, mais peut-être aussi par convention, nous emploierons la prononciation japonaise dans cet article, même si la plupart des textes cités sont chinois. Il en sera de même pour les kōan, en chinois gong'an. 2. "A special transmission outside the scriptures,/ Not founded upon words and letters;/ By pointing directly to [one's] mind /It lets one see into [one's own true] nature and [thus] attain Buddhahood." Tous les extraits tirés d'ouvrages en anglais et cités dans cet article ont été traduits par nous.

3. Dans son article, Christine Young-Merllié cite en exergue Chenching Ke-wen: «En ce qui concerne le zen, l'expérience est tout. Tout ce qui n'est pas basé sur l'expérience est extérieur au Zen" (1996: 273). 4. Le terme zazen vient du chinois zuo chan (坐禪) qui signifie littéralement: «méditation assise».

5. Le terme kōan vient du mot chinois gong'an (公案) qui signifie «archives publiques». Ce terme appartenant au vocabulaire juridique renvoie aux archives où sont consignés des "cas" pouvant servir de jurisprudence. De même, les kōan réunis dans les recueils zen obéissent au même principe: ils «ne représentent pas l'opinion personnelle d'un seul homme, mais plutôt le principe supérieur, reçu pareillement par nous et par les centaines et les milliers de bodhisattvas des trois royaumes et des dix directions. Ce principe s'accorde avec la source spirituelle, correspond au sens mystérieux, détruit la naissance-et-lamort et dépasse les passions.» (Citation du Zhong-fen Ho-shang Kuang-lu empruntée à Ruth Fuller-Sasaki [1985: 258]).

6. "Your attitude toward the verbal teachings of the buddhas and the patriarchs must be as hostile as that toward a deadly enemy."

7. De fait, ces mêmes maîtres qui dénigrent les Écritures révèlent dans certains dialogues une connaissance et une compréhension très profondes de ces textes; depuis Bodhidharma lui-même, qui serait entré en Chine avec un exemplaire du Lankāvatāra Sūtra, jusqu'à Huineng, le sixième et dernier patriarche officiel du zen ou chan chinois, qui n'a jamais caché son attachement pour le Vajracchedikā Sütra (Sütra du Diamant).

8. "Once, one of the monk of Geat Teacher Kōtaku of Mt. Ungo was reading a sutra in his room. The master called out through the window, 'What sūtra are-you reading?' The monk answered, 'The Vimalakirti-nirdesa Sütra.' The master said, 'I didn't ask about the sutra in your hands. What sutra are-you reading?' The monk was suddenly enlightened."

9. "The kōan urges us to abandon our rational thought structures and step beyond our usual state of consciousness in order to press into new and unknown dimensions."

10. Le texte qui introduit ce sermon nous apprend que c'est à la demande du gouverneur Wang et de ses fonctionnaires que Lin-Tsi était monté en chaire (voir Demiéville, 1972: 21).

11. Ce que Demiéville rend par "khät» est le caractère chinois he (喝, katsu en japonais). Impossible à traduire, c'est à la fois un cri, une interjection et, nous dit le Dictionnaire classique de la langue chinoise (1966: 145), «un son guttural, rauque et étouffé ».

12. Un des exemples les plus célèbres est un des kōan recueillis dans le Bi Yan Lu sous le titre "Fu Da-shi se prononce sur le Sütra»: «L'empereur Wu des Liang invita Fu Da-shi à prononcer un sermon sur le Sütra du Diamant. Da-shi monta sur la chaire, frappa un coup sur la table et descendit. L'empereur Wu fut stupéfait. Zhi-gong demanda: "Sire, avez-vous compris? "L'empereur répondit: "Non, je ne comprends pas." Zhi-gong dit: "Da-shi a terminé son sermon" " (Bi Yan Lu, cas 67 [Cleary, 1977 : 376; NT]: "Emperor Wu of Liang asked Fu Da-shi to give a lecture on the Diamond Sutra. Fu Da-shi mounted the platform, struck the reading desk with his baton, and descended from the platform. The emperor was dumbfounded. Zhi-gong said to him, 'your Majesty, have you understood?' The emperor said, 'No, I do not understand.' Zhi-gong said, 'Da-shi has concluded his lecture'”). 13. Qu'on pense par exemple à Zhao-zhou qui répondait à la question de la vérité du Bouddha de la manière suivante: «un cyprès dans la cour" (Wu Men Guan, cas 37).

14. "What is Zen? - It is this. - What is the Way? - Yes."

15. Poème conclusif du cas $37 \mathrm{du}$ Wu Men Guan: "Words cannot express things; / Speech does not apply. / Swayed by words, one is lost;/ Blocked by phrases, one is bewildered."

16. Autrement dit, l'adjectif en chinois est un verbe: $d a$ (大), "grand", doit se traduire plus exactement "être grand", et la proposition ne se construira pas sous le schéma "A est grand", mais bien "A da".

17. Bi Yan Lu, cas 2: "Jōshū [Zhao-zhou] spoke to the assembly and said, 'The real Way is not difficult. It only abhors choice and attachment. With but a single word there may arise choice and attachment'." 18. Le maître zen japonais Musō Soseki pourra ainsi écrire: «J'ai jeté cette toute petite chose qu'on appelle "Moi" et je suis devenu le monde immense» (Shibata, 1993: 36).

19. "Kōans utilize decentric signs and signifiers - or it could be said that the kōans themselves represent the process of the decentring of all signs - so that they function flexibly and provisionally as symbolic discourse without reference to an absolute or transcendental Signified." 20. "In the oneness of the void the two are one,/And each of the two contains in itself all the ten thousand things."

21. Nāgārjuna, Müla-Madhyamakakārikā, chap.XXIV: "It is a truth of worldly convention."

22. Le paradoxe se donnant comme un au-delà ou un opposé ( $\pi \alpha \rho \alpha ́)$ d'un

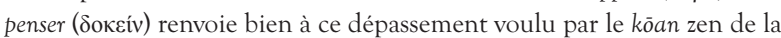
conscience discriminante, de la pensée ratiocinante.

23. C'est ce que Thomas Cleary (1977), dans sa traduction du Bi Yan Lu (introduction du cas 70), traduit par «immediacy (immédiateté) » que Katsuki Sekida traduit ainsi dans sa version du Bi Yan Lu (1977: 332).

24. Wu Men Guan, cas 6: "Once in ancient times, when the WorldHonored One was at Mount Grdhrakūta, he held up a flower before his assembly. They all were silent. Only Mahākāshyapa broke into a smile. The World-Honored One said: 'I have the True Eye of the Dharma, the Marvelous Mind of Nirvana, the True Form of No-Form, the Subtle Gate of the Dharma which does not depend on any word or letter, but is a special transmission outside the scriptures. With this, I entrust Mahākāshyapa'."

25. "This coming to a cul-de-sac is really the true starting point in the study of Zen." 


\section{RÉFÉRENCES BIBLIOGRAPHIQUES}

AitKen, R. [1991]: The Gateless Barrier, New York, North Point Press. App, U. [1994]: Master Yun Men, New York, Kodansha International. ARISTOTE [1990]: Topiques, Paris, Vrin.

Beaufret, J. [1974]: Dialogue avec Heidegger, vol.3, Paris, Minuit. Cheng, A. [2004]: Entretiens de Confucius, Paris, Seuil.

ClearY, T. [1977]: The Blue Cliff Record, Boston, Shambhala.

Couvreur, F.S. [1966]: Dictionnaire classique de la langue

chinoise,Taïwan, Kuangchi Press.

Deleuze, G. [1969]: Logique du sens, Paris, Minuit.

DemiÉVILLE, P. [1972]: Entretiens de Lin-Tsi, Paris, Fayard.

Dōgen Zenjı [1977]: Shōbōgenzō. The Eye and Trasury of the True Law,

Tokyo, Nakayama Shobō.

Dumoulin, H. [1988] : Zen Buddhism: a History, New York, Macmillan

Publishing Co.

FA-HAI [1995]: Le Soûtra de l'Estrade du Sixième Patriarche Houeineng,

Paris, Seuil.

FONG, Y.-L. [1992]: Précis d'histoire de la philosophie chinoise, Paris, Le

Mail.

FUlLER-SASAKI, R. [1985]: «L'usage du koan en Chine », dans Tch'an-

Zen, Racines et Floraisons, Paris, Les Deux Océans, coll. «Hermès 4 ", 257-267.

GARFIELD, J. L. [1995]: The Fundamental Wisdom of the Middle Way,
Nāgārjuna’s Mülamadhyamakakārikā, New York, Oxford University Press. GeRnet, J. [1977]: Entretiens du Maître de Dhyāna Chen-houei, Paris, Publications de l'École française d'Extrême-Orient, vol.XXXI. Granet, M. [1968] : La Pensée chinoise, Paris, Albin Michel. HeInE, S. [1994]: Dōgen and the Kōan Tradition, New York, State University of New York Press.

IsshÜ, M. [1965]: The Koan Zen, New York, Harcourt Brace \& Co. Jullien, F. [1995]: Le Détour et l'accès, stratégies du sens en Chine, en Grèce, Paris, Grasset.

LACAN, J. [1990]: Séminaire VII : éthiques de la psychanalyse, Paris, Seuil. MerLeau-Ponty, M. [1968]: Résumés de cours, Paris, Gallimard. Merton, T. [(1961) 1995]: Mystique et Zen, Paris, Albin Michel. PLATON [1989]: Cratyle, Paris, Gallimard.

SEKIDA, K. [1977]: Two Zen Classics, New York et Tokyo, Weatherhill. SENG Ts'An [1992]: Hsin Hsin Ming, Paris, Arfuyen.

Shibata, M. et M. Shibata [1993]: Sermons sur le Zen, Paris, Albin Michel.

SuZUKI, D.T. [1964]: An Introduction to Zen Buddhism, New York, Grove Press;

[1972]: Essais sur le Bouddhisme Zen, 3 vol., Paris, Albin Michel. YOUNG-MERLLIÉ, C. [(1972) 1996]: «La mystique des maîtres de Tch'an ", dans M.-M. Davy, Encyclopédie des mystiques, tome IV, Paris, Payot, 293-297. 